\title{
Influencia de niveles de infestación de Tubixaba tuxaua (Monteiro \& Lordello, 1980) en el cultivo de soja
}

\author{
Influence of levels of Tubixaba tuxaua infestation (Monteiro \& \\ Lordello, 1980) in the cultivation of soybean crop
}

\author{
Carine Andressa Kolling ${ }^{1 *}$ y Marco Maidana Ojeda ${ }^{1,2}$ \\ ${ }^{1}$ Universidad Católica Nuestra Señora de la Asunción, Facultad de Ciencias Agropecuarias, Unidad Pedagógica María \\ Auxiliadora. Tomás Romero Pereira, Paraguay. \\ ${ }^{2}$ Centro de Desarrollo e Innovación Tecnológica. Hohenau, Paraguay.
}

*Autor para correspondencia: carinekolling@gmail.com

Conflicto de interés: Los autores declaran no tener conflicto de interés.

\section{Licencia:}

Artículo publicado en acceso abierto con una licencia Creative Commons CC-BY

Historial:

Recibido: $11 / 10 / 17$;

Aceptado: 01/07/19

Periodo de Publicación: Julio-Diciembre de 2019

\section{RESUMEN}

Tubixaba tuxaua es un fitonemátodo que ocasiona daños a los cultivos y fue recientemente identificado en muestras de suelo del Paraguay. Debido a la escasez de informaciones locales de los daños ocasionados al cultivo de soja, se realizó un estudio con el objetivo de evaluar los efectos sobre el desarrollo y rendimiento ocasionados por distintas densidades de Tubixaba tuxaua en el cultivo de soja. El experimento se realizó en condiciones de campo, en la zafra 2016/2017, en Tempiaporenda, Caazapá, Paraguay, en una parcela con 10 años de producción de soja. De acuerdo a los síntomas observados a los 48 días después de la siembra se delimitaron 3 áreas de infestación y fueron distribuidas en un diseño experimental completamente al azar con 12, 6 y 7 repeticiones, para las áreas de infestación alta, medio y libre de Tubixaba tuxaua respectivamente. Las variables analizadas fueron; población del nemátodo, área foliar, masa seca, altura de planta, número de vainas, peso de 100 granos y rendimiento. Los resultados demostraron diferencias estadísticas entre los tratamientos para todas las variables a excepción de peso de 100 granos. Se observó que en las áreas de mayor población de nematodos provocó una disminución de la altura de planta, número de vainas, área foliar, masa seca y rendimiento con una tendencia decreciente.

Palabras clave: Glycine max, Aporcelaimidae, ectoparásito, fitonemátodo.

\begin{abstract}
Tubixaba tuxaua is a phytonematode that causes damage to crops and was recently identified in soil samples from Paraguay. Due to the scarcity of local information on the damage caused to soybean cultivation, a study was carried out with the objective of evaluating the effects on development and yield caused by different densities of Tubixaba tuxaua in the soybean crop. The experiment was carried out in field conditions, in the 2016/2017 cycle, in Tempiaporenda, Caazapá, Paraguay, in a plot with 10 years of soybean production. According to the symptoms observed 48 days after sowing, 3 areas of infestation were delimited and distributed in a completely randomized experimental design with 12, 6 and 7 repetitions, for the areas of high, medium and free infestation of Tubixaba tuxaua respectively. The variables analyzed were; population of the nematode, foliar area, dry mass, height of plant, number of pods, weight of 100 grains and yield. The results showed statistical differences between the treatments for all the variables except weight of 100 grains. It was observed that in areas with a greater population of nematodes, there was a decrease in plant height, number of pods, foliar area, dry mass and yield with a decreasing tendency.
\end{abstract}

Key words: Glycine max, Aporcelaimidae, ectoparasit, phytonematode.

\section{INTRODUCCIÓN}

La soja constituye uno de los principales rubros agrícolas de importancia económica para el Paraguay. En el ranking mundial, Paraguay es el sexto mayor productor de soja con una producción estimada de 8,80 millones de toneladas y el cuarto mayor exportador con 4,60 millones de toneladas en la zafra 2015-2016 (CAPECO, 2016).

El cultivo de soja puede ser afectado por fitonemátodos como Meloidogyne javanica, Pratylenchus coffeae y Rotylenchulus reniformis y Heterodera glycines (Centurión, Uehara, Kanno, 
Baigorri y Shimizu, 2001; Centurión, Shimizu \& Momota, 2004). Los nematodos fitoparásitos están adquiriendo cada vez mayor importancia, constituyéndose en una seria amenaza a la sanidad de los cultivos de importancia económica (McCarter, 2009), requiriendo un cambio drástico en el manejo, pasando del uso intensivo del control químico al empleo de estrategias más holísticas, en las cuales una amplia combinación de medidas de control es empleada (Sikora, Bridge \& Starr, 2005).

Leiva, Severino é Zabini (2016) en los años 20152016 a partir 295 de muestras de suelos analizadas provenientes de diferentes localidades de la región Oriental del Paraguay, identificaron la presencia de quince géneros de nematodos, entre ellas Tubixaba sp. en un $16 \%$, siendo el primer reporte de este género en Paraguay.

Tubixaba tuxaua es un nematodo ectoparásito de hábito polífago perteneciente al Orden Dorylaimida y a la Familia Aporcelaimidae (Vovlas, Lambertini, Antonio, Monteiro \& Sharma, 1987), reportado por primera vez en Marechal Cândido Rondon, Paraná, Brasil, asociado a plantas de soja con síntomas de enanismo (Monteiro \& Lordello, 1980).

Los nematodos del género Tubixaba spp. se caracterizan por presentar cuerpo muy largo, cutícula espesa con líneas cruzadas en la capa cortical, apertura oral hexagonal, estilete axial con la apertura ocupando una tercera parte de su longitud (Monteiro \& Lordello, 1980). Vovlas et al. (1987) realizaron una descripción más detallada de la morfología de esa nueva especie, comprobando su gran tamaño, con una longitud promedio para $T$. tuxaua de $11,3 \mathrm{~mm}$ para machos y $11,8 \mathrm{~mm}$ para hembras, el odontoestilete, presenta un largo promedio de $31 \mu \mathrm{m}$.

La ocurrencia del nematodo está directamente relacionada con el escaso desarrollo de las plantas, reducción de la altura, clorosis y disminución de la producción (Carneiro e Carneiro, 1983). T. tuxaua se reportó como causante de reducción de rendimientos en los cultivos de soja, maíz, trigo y mandioca (Carneiro e Carneiro, 1983; Roese, Romani, Furnaletto, Stangarlin é Portz, 2001).

Ensayos a campo y en macetas demostraron que $T$. tuxaua reduce la altura, masa verde y masa seca en abonos verdes como Crotalaria juncea, Mucuna aterrima, Mucuna deeringiana y Canavalia ensiformis, utilizados en rotación de verano con cultivos económicos en el oeste de Paraná, Brasil (Furlanetto et al., 2008). Abonos verdes de invierno han sido evaluados como alternativa de control y susceptibilidad al nematodo. Los resultados demostraron que todos los materiales evaluados fueron susceptibles, pero hubo reducción de la población de nematodos con nabo forrajero, lo cual puede ser una alternativa a utilizar en rotación para el manejo de T. tuxaua (Furlanetto, Cares, Cruz \& Guimarães, 2012).

En estudios realizados en parcelas con $T$. tuxaua, se observaron que después de la siembra de trigo hubo mayor densidad poblacional (20 a 115 nematodos/300 cc de suelo) del nematodo en comparación en aquellas cultivados con soja (20 a 54 nematodos/300 cc de suelo) y maíz (18 a 23 nematodos/300 cc de suelo), además de una reducción de rendimientos de 50 a $70 \%$ para el trigo, 75 a $76 \%$ para soja y de $46 \%$ para el maíz (Furlanetto et al., 2010).

Debido a la escasez de informaciones nacionales de los daños ocasionados por ese nematodo fitoparásito, el trabajo fue realizado con el objetivo de evaluar los efectos sobre el desarrollo y rendimiento ocasionados por distintas densidades de Tubixaba tuxaua sobre el cultivo de soja en condiciones de campo.

\section{MATERIALES Y MÉTODOS}

El ensayo se llevó a cabo en una parcela agrícola con 10 años de producción de soja con diagnóstico de la presencia del nematodo $T$. tuxaua, en la localidad de Tempiaporenda, distrito de Tava'i, Caazapá, Paraguay. El experimento a campo se realizó en la zafra de soja 2016-2017 durante los meses de septiembre 2016 a marzo 2017. La textura del suelo del área presenta niveles de $26,14 \%$ de arena, $26,41 \%$ de limo y $47,45 \%$ de arcilla. El cultivo procedente al experimento fue Crotalaria juncea.

El manejo del cultivo desde la siembra hasta la cosecha fue realizado por el propietario de la finca de acuerdo a las necesidades de la planta a excepción del control de nematodos. La siembra se realizó el día 10 de septiembre de 2016 y el material experimental utilizado fue la variedad de soja M6410 IPRO. Previo a la instalación del experimento, se determinaron en base a los síntomas descritos por el área libre y en el área infestada por T. tuxaua (Carneiro e Carneiro, 1983) 
y se realizó análisis nematológico y químico del suelo, los análisis fueron realizados por el Laboratorio de Suelos y Consultoría "Agronómico", de la ciudad de Hernandarias, Alto Paraná.

La cuantificación de la población de $T$. tuxaua se realizó antes de la siembra y en el estadío R7 (Fehr, Caviness, Burmood \& Pennington, 1971) en el laboratorio de la Universidad Católica, Unidad Pedagógica María Auxiliadora, Tomás Romero Pereira, Paraguay, siguiendo la metodología descrita por Furlanetto et al., (2012) que consiste en: tomar $200 \mathrm{~cm}^{3}$ de suelo de cada muestra, homogeneizar con agua en baldes de plástico, pasar la mezcla por tamices de 20 y 60 mesh, respectivamente. El residuo del tamiz fue colocado en vaso de precipitado y depositado en placa de Petri para la cuantificación e identificación en estereoscopio. La identificación de $T$. tuxaua fue realizada mediante los caracteres morfológicos, descritos por Monteiro \& Lordello (1980) y Vovlas et al. (1987).

A los 48 días después de la siembra se realizó la demarcación de las unidades experimentales, de acuerdo a los síntomas observados (altura de planta y área foliar) fueron agrupados en tres niveles de infestación por el nematodo: área de infestación alta (manchón con plantas de menor altura y área foliar); área de infestación media (bordes del manchón) y área libre (plantas desarrolladas normalmente), estos fueron distribuidos en un diseño experimental completamente al azar con 12, 6 y 7 repeticiones para infestación alta, media y libre de $T$. tuxaua respectivamente. Cada unidad experimental estaba constituida por 4 hileras de 5 metros. El área útil fueron las dos hileras centrales de cada unidad experimental $\left(4,5 \mathrm{~m}^{2}\right)$.

Las variables evaluadas y las metodologías utilizadas fueron las siguientes:

Altura de planta: la medición se realizó con cinta métrica desde la base del tallo hasta el ápice de la rama con vaina superior, en 10 plantas de cada unidad experimental, en el momento de madurez fisiológica. El resultado fue expresado en $\mathrm{cm}$.planta-

1. Número de vainas: se efectuó el conteo del número de vainas en 10 plantas de cada unidad experimental, en el momento de madurez fisiológica. El resultado se promedió en vainas.planta-1 ${ }^{-1}$ Área foliar: el área foliar se analizó en el estadio R3 de la etapa reproductiva del cultivo. Fueron extraídas 2 plantas por cada unidad experimental. Se utilizó el Software Easy Leaf Area (Easlon y Bloom, 2014). El resultado fue expresado en $\mathrm{cm}^{2}$. planta-1. Materia seca: la materia seca se analizó en el estadio R3 de la etapa reproductiva del cultivo. Fueron extraídas 2 plantas por cada unidad experimental. La masa seca se obtuvo mediante el secado en estufa a $60^{\circ} \mathrm{C}$ hasta adquirir masa constante. El resultado fue expresado en g.planta-1.

Peso de 100 granos: se contabilizó 100 granos del área útil de cada unidad experimental, éstos fueron pesados mediante una balanza de precisión de 0,001 gramos y ajustados a $14 \%$ de humedad. El resultado fue expresado en gramos (g). Rendimiento: los granos obtenidos del área útil de cada unidad experimental fueron pesados en una balanza de precisión. El resultado obtenido fue expresado en $\mathrm{kg} \cdot \mathrm{ha}^{-1}$, por medio de la fórmula: Rendimiento $\left(\mathrm{kg} \cdot \mathrm{ha}^{-1}\right)=$ peso del área útil×10000)/área úti* $\left(\left(100-\mathrm{H}^{\circ}\right) / 86\right)$.

Los datos obtenidos fueron sometidos al análisis de varianza y test de Tukey al $5 \%$ de probabilidad de error mediante el programa estadístico InfoStat ( $\mathrm{Di}$ Rienzo et al., 2016). Además, se realizó análisis de regresión entre la densidad poblacional de nematodo en R7 (variable predictora) con la altura de planta, área foliar, masa seca, número de vainas por planta, peso de 100 granos y rendimiento.

\section{RESULTADOS Y DISCUSIÓN}

Los síntomas observados en el cultivo fueron plantas subdesarrolladas (Figura 1D.), semejante a las características descritas por Monteiro \& Lordello (1980), Carneiro y Carneiro (1983). El análisis nematológico evidenció la presencia de Helicotylenchus spp. ( 80 nematodos $/ 200 \mathrm{~cm}^{3}$ ) en el área libre y Helicotylenchus spp. (48 nematodos $/ 200 \mathrm{~cm}^{3}$ ) y Pratylenchus spp. (100 nematodos $/ 200 \mathrm{~cm}^{3}$ ) en el área infestada por $T$. tuxaua, considerados como niveles críticos bajos (Niblack, 2005; Niblack, 2009). Según Tihohod (1993), las plantas raramente están sujetas a la influencia de un único patógeno potencial, especialmente por la gran cantidad de patógenos que viven en el suelo. En un trabajo realizado por Furlanetto et al. (2010), áreas infestadas con $T$. tuxaua estaban asociadas a la presencia de Helicotylenchus y Scutellonema en poblaciones bajas.

La población de $T$. tuxaua en el área de infestación alta fueron de 47 a 101 nematodos $/ 200 \mathrm{~cm}^{3}$ en el inicio del estudio con un promedio de 61 nematodos $/ 200 \mathrm{~cm}^{3}$, mientras que en el área de infestación media la población de nematodo varió de 0 a 54 nematodos $/ 200 \mathrm{~cm}^{3}$ con un promedio de 15 nematodos $/ 200 \mathrm{~cm}^{3}$. Para la evaluación que se 
realizó en estadío $\mathrm{R} 7$, la población de nematodo en el área de infestación alta fue de 30 a 52 nematodos/200 $\mathrm{cm}^{3}$ con un promedio de 42,42 espécimen $/ 200 \mathrm{~cm}^{3}$ de suelo, y en el área de infestación media fue de 1 a 22 nematodos/200 $\mathrm{cm}^{3}$, con un promedio de 8,83 espécimen $/ 200 \mathrm{~cm}$ de suelo, observándose diferencias significativas entre los promedios del área de infestación alta y media. Según Furlanetto et al. (2010) densidades poblacionales de 54 especímenes $/ 300 \mathrm{~cm}^{3}$ pueden llegar a causar pérdidas de 75 a $76 \%$ en la producción de granos de soja.

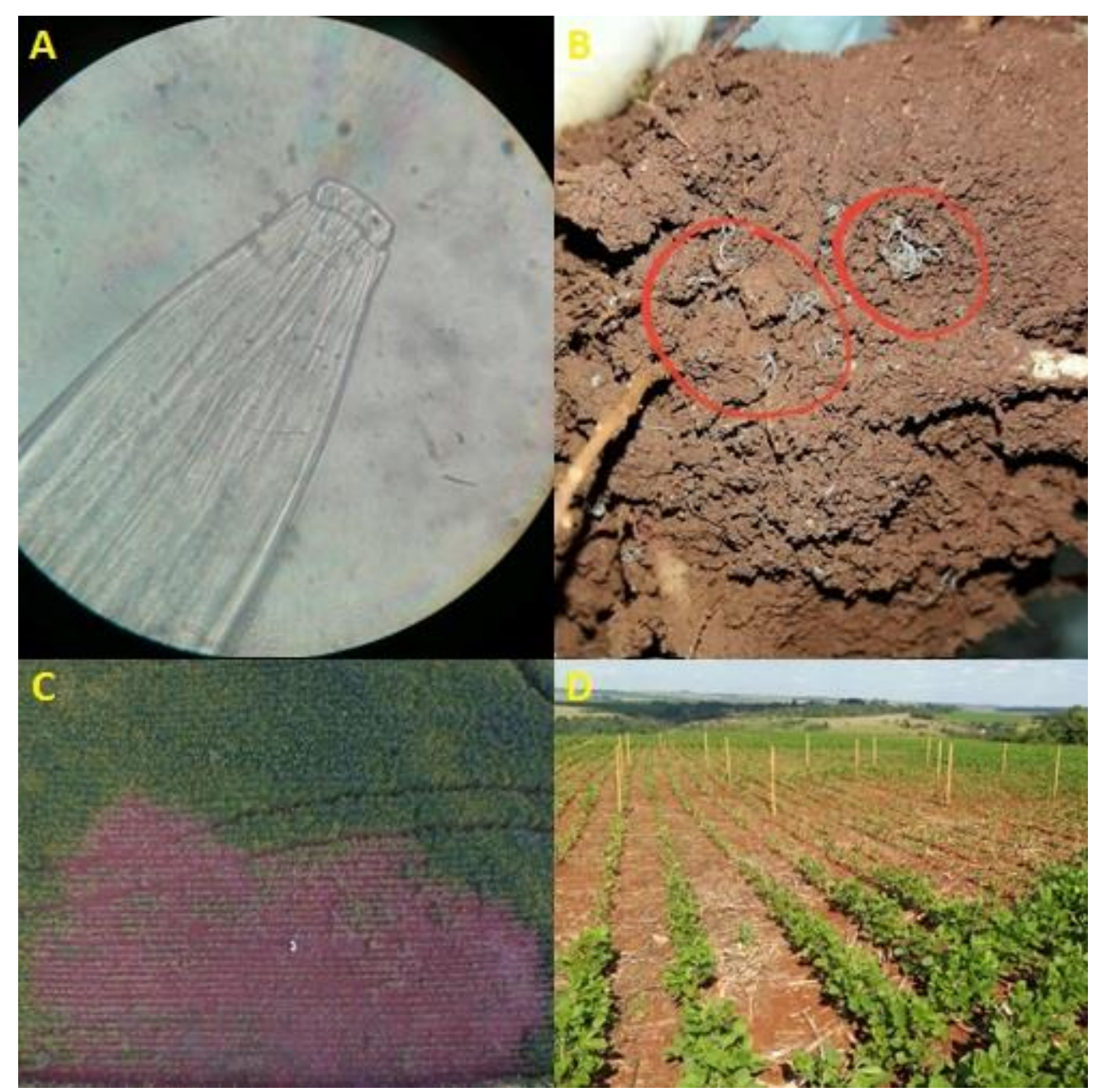

Figura 1. A. Región anterior de T. tuxaua mostrando la apertura oral. B. Especímenes de T. tuxaua sobre raíz de una planta. C. Área infestada por el nematodo. D. Manchón de plantas subdesarrolladas a consecuencia del fitoparásito. Zafra 2016/2017. Caazapá, Paraguay.

La altura de planta se observó una reducción de $62,5 \%$ en el área de infestación alta en comparación con el área libre (Tabla 1). El porcentaje de reducción demostrado por Furlanetto et al. (2010), fue de $54 \%$ para la altura de plantas, en poblaciones de 20 a 54 nematodos $/ 300 \mathrm{~cm}^{3}$ de suelo. El coeficiente de correlación demostró una estrecha relación negativa entre altura de planta y población (Figura 2A). Un incremento en la población de $T$. tuxaua causa reducciones significativas en el crecimiento de la planta. Dildey et al. (2011), demostraron una correlación negativa entre altura de planta y densidad poblacional de $T$. tuxaua con $\mathrm{R}^{2}=0,9996$, observaron una reducción de aproximadamente $27 \%$ en el crecimiento en altura de la planta de soja en una población de 80 nematodos.

Para el área foliar ocurrió una reducción de 95,6\% en el área de infestación alta (Tabla 1) en 
comparación con el área libre de T. tuxaua. El análisis de regresión indicó alta correlación entre ambas variables (Figura 2C.), demostrando que al haber un aumento en la población de $T$. tuxaua ocurre una reducción significativa en el área foliar de las plantas. El área foliar en poblaciones de 30 a 50 nematodos $/ 200 \mathrm{~cm}^{3}$ de suelo demostró poca variación (Figura 2C.). La masa seca del área de alta infestación fue de 2,69 g.planta-1 (Tabla 1), hubo una reducción de $91,9 \%$ en el área de infestación alta (Tabla 1) en comparación con el área libre. El incremento de la población de $T$. tuxaua causó reducciones significativas en la masa seca de las plantas.

Tabla 1. Efecto del nematodo T. tuxaua sobre parámetros fisiológicos y rendimiento de soja. Zafra 2016/2017. Caazapá, Paraguay.

\begin{tabular}{|c|c|c|c|c|c|c|c|}
\hline Tratamientos & $\begin{array}{l}\text { Población } \\
\text { (espec. } 200 \\
\mathrm{~cm}^{3} \mathrm{de} \\
\text { suelo) }\end{array}$ & $\begin{array}{c}\text { Altura de } \\
\text { planta }(\mathrm{cm})\end{array}$ & $\begin{array}{c}\mathrm{N}^{\circ} \\
\text { vainas.planta-1 }\end{array}$ & $\begin{array}{c}\text { Área foliar } \\
\left(\mathrm{cm}^{2} \cdot \text { planta }^{-1}\right)\end{array}$ & $\begin{array}{l}\text { Masa seca } \\
(\text { g.planta-1) }\end{array}$ & $\begin{array}{c}\text { Peso de } \\
100 \text { granos } \\
(\mathrm{g})\end{array}$ & $\begin{array}{c}\text { Rendimiento } \\
\left(\mathrm{kg} \cdot \mathrm{ha}^{-1}\right)\end{array}$ \\
\hline Infest. Alta & $42,42 \mathrm{a}$ & $37,74 a$ & $18,28 \mathrm{a}$ & $186,08 \mathrm{a}$ & $2,69 a$ & 16,20 & 1299,28 a \\
\hline Infest. Media & $8,83 \mathrm{~b}$ & $78,32 b$ & $78,52 b$ & $1916,24 b$ & $16,74 b$ & 16,74 & $4630,43 b$ \\
\hline Área libre & $0,00 \mathrm{~b}$ & $100,56 \mathrm{c}$ & $105,01 \mathrm{c}$ & $4266,19 \mathrm{c}$ & $33,32 \mathrm{c}$ & 16,89 & $5462,73 b$ \\
\hline Promedio & 22,48 & 65,07 & 57,02 & 1743,75 & 14,64 & 16,52 & 3264,52 \\
\hline Fc & $94,78 * *$ & $55,19 * *$ & $54,35 * *$ & $90,29 * *$ & $84,88 * *$ & $3,17^{\mathrm{NS}}$ & $93,73 * *$ \\
\hline CV (\%) & 31,37 & 20,07 & 32,32 & 36,67 & 33,91 & 3,79 & 21,39 \\
\hline
\end{tabular}

En la columna, medias con la misma letra minúscula no presentan diferencias significativas por el test de Tukey al $5 \%$ de probabilidad de error $(p>0,05)$.

Para la variable número de vainas hubo diferencias significativas, con una reducción de $82,6 \%$ en el número de vainas del área de alta infestación (Tabla 1). De acuerdo a Furlanetto et al. (2010), áreas infestadas con $T$. tuxaua presentaron una reducción de 58 a 73\% para el número de vainas en soja en poblaciones de 20 a 54 nematodos $/ 300 \mathrm{~cm}^{3}$ de suelo. Los resultados demostraron que en áreas con mayor población del nematodo hubo menor número de vainas por plantas de soja (Figura 2B.). Dildey et al. (2011a), demostraron una correlación negativa entre número de vainas y densidad poblacional de $T$. tuxaua con $\mathrm{R}^{2}=0,9738$, indicaron una reducción de aproximadamente $70 \%$ en el número de vainas por planta de soja con una población de 80 nematodos/8 litros de suelo.

No se observó diferencias significativas para el peso de 100 granos de soja (Tabla 1), a partir de ello, se deduce que el nematodo no afectó el peso de los granos, sin embargo, Furlanetto et al. (2010) observaron perjuicios cualitativos en los granos de soja de áreas infestadas con T. tuxaua, en el cual, las vainas habían presentado mayor incidencia de granos chuzos y/o pequeños. Esto se debe a que los componentes de rendimiento como número de vainas por planta y peso de 100 granos son afectados de acuerdo al momento de ocurrencia del estrés en el cultivo de soja, viéndose afectado el número de vainas cuando el estrés ocurre antes de R4 y el peso de 100 granos cuando el estrés ocurre en el llenado de granos (R5-R6) (Kantolic, Giménez y De la Fuente, 2003), en este caso los síntomas por T. tuxaua fueron observados a partir de $\mathrm{Vn}$ (48 días después de siembra).

El rendimiento entre el área libre y el área de infestación media no presentaron diferencias estadísticas significativas entre sí, pero ambos difieren significativamente del área de infestación alta. El promedio del rendimiento del área de infestación alta fue de 1299,28 kg.ha-1 (Tabla 1), comparado con el área libre, ocurrió una reducción de $76 \%$, estos resultados conciertan con Furlanetto et al. (2010), quienes demostraron que áreas infestadas con $T$. tuxaua presentaron niveles de daño en soja de 75 a $76 \%$ para la producción de granos. 

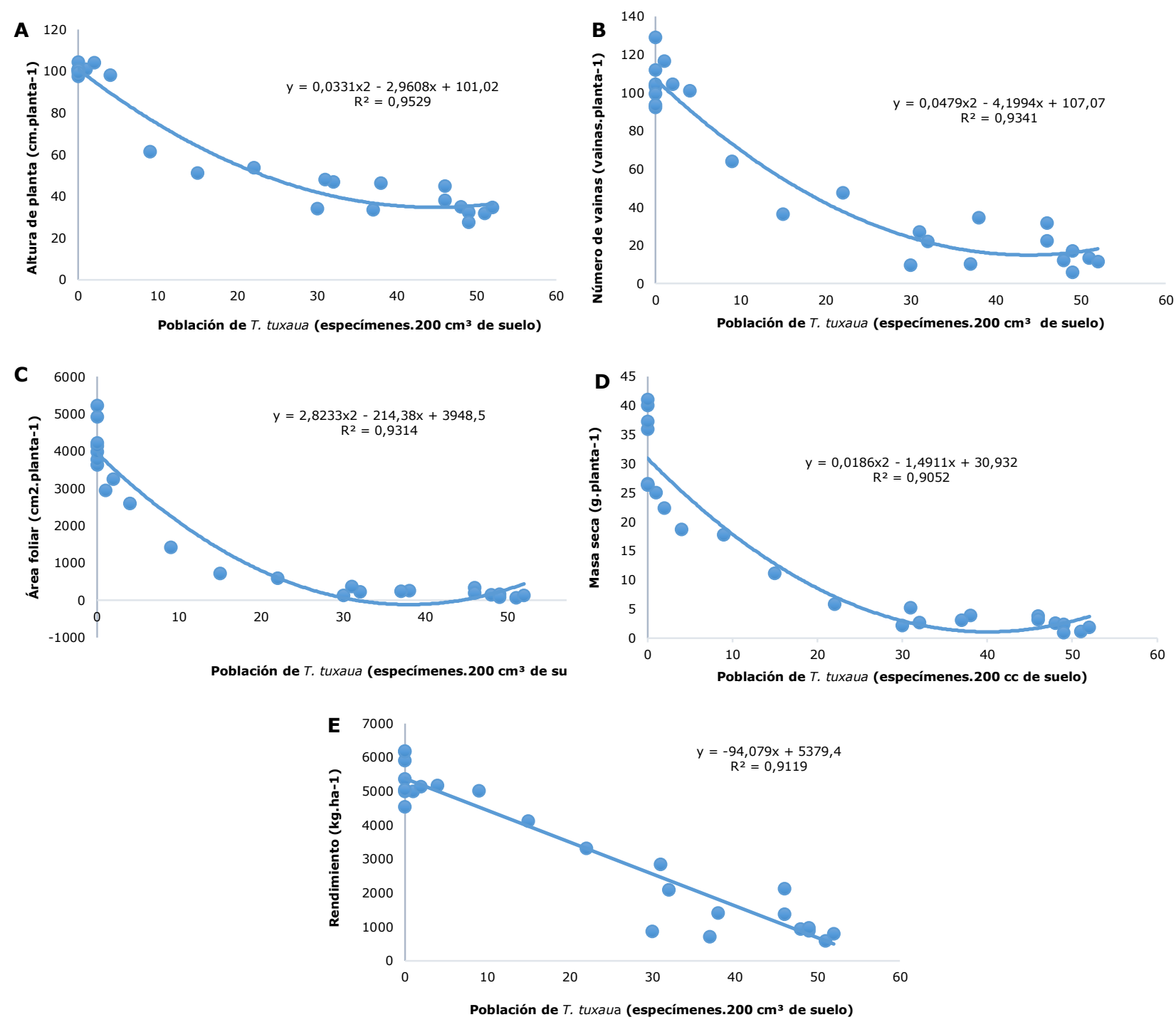

Figura 2. Análisis de regresión de las variables. A. altura de planta B. número de vainas C. área foliar D. masa seca $\mathbf{E}$. rendimiento, en relación al número de nematodos $/ 200 \mathrm{~cm}^{3}$ de suelo. Zafra 2016/2017. Caazapá, Paraguay.

\section{CONCLUSIONES}

El nematodo $T$. tuxaua afecta el cultivo de soja, generando reducciones de $62,5 \%$ para la altura de planta, $82,6 \%$ para el número de vainas, $95,6 \%$ para el área foliar, $91,9 \%$ para la masa seca, y $76 \%$ para el rendimiento, con una tendencia decreciente a medida que aumenta las poblaciones de nematodos.

El peso de 100 granos no es afectado por altas poblaciones de $T$. tuxaua.

\section{REFERENCIAS BIBLIOGRÁFICAS}

CAPECO (Cámara Paraguaya de Exportadores y Comercializadores de Cereales y Oleaginosas).
(2016). Ranking mundial. Consultado 05 sep. de 2016. Disponible en http://capeco.org.py/rankingmundial-es/

Carneiro, R.M.D.G. e Carneiro, R.G. (1983). Estudos preliminares sobre o nematoide Tubixaba tuxaua Monteiro \& Lordello, 1980, na cultura do trigo no sudoeste do Paraná. Sociedade Brasileira de Nematologia, 7, 251-259.

Centurión, F., Shimizu, K. \& Momota, Y. (2004). First record of soybean cyst nematode, Heterodera glycines Ichinohe from Paraguay. Nematological Research, 34, 39-42.

Centurión, F., Uehara, T., Kanno, T., Baigorri, H. y Shimizu, K. (2001). Nematodos parásitos em cultivos de soja y pasturas en Argentina, Brasil y 
Paraguay y su control. Nematologia Brasileira, 25 (Supl.): 107

Di Rienzo J.A., Casanoves, F., Balzarini, M.G. Gonzalez, L., Tablada, M. y Robledo, C.W. (2016). InfoStat versión 2016. Grupo InfoStat. Argentina: FCA, Universidad Nacional de Córdoba. Disponible en : http://www.infostat.com.ar

Dildey, O.D.F., Broetto, L., Müller, M.A., Mioranza, T.M., Coltro, S., Meinerz, C.C... Bonett, L.P. (2011). Influência de diferentes populações de Tubixaba tuxaua na altura de planta de soja (Glycine max (L.) Merril). Tropical Plant Pathology (BR), 36, 1215.

Dildey, O.D.F., Broetto, L., Müller, M.A., Mioranza, T.M., Coltro, S., Meinerz, C.C....Bonett, L.P. (2011a). Influência de diferentes populações de Tubixaba tuxaua no número de vagens em soja (Glycine max (L.) Merril). Tropical Plant Pathology (BR), 36, 1217.

Easlon, H.M. \& Bloom, A.J. (2014). Easy Leaf Area: Automated digital image analysis for rapid and accurate measurement of leaf area. Applications in plant sciences, 2(7), 1400033

Fehr, W.R., Caviness, C.E., Burmood, D.T. \& Pennington, J.S. (1971). Stage of development descriptions for soybeans (Glycine max L.). Crop science, 11(6), 929-931

Furlanetto, C., Cares, J.E., Cruz, M.I.F. \& Guimarães, R. (2012). Development of winter cover crops in soil infested with the nematode Tubixaba tuxaua in Western Paraná, Brazil. Rev. Nematropica. 42(2): 314-319.

Furlanetto, C., Davi, J.J.S., Grabowski, M.M.S., Dias-Arieira, C.R., Layter, N.A. \& Seifert, K. (2008). Reação de adubos verdes de verão ao nematoide Tubixaba tuxaua. Tropical Plant Pathology (BR), 33 (6), 403-408.

Furlanetto, C., Seifert, K.E., Fensterseifer, C.E., Page, E.C., Davi, J.J.S. é Grabowski, M.M.S. (2010). Desenvolvimento das culturas de soja, milho e trigo cultivadas em áreas infestadas com o nematóide Tubixaba tuxaua no Oeste do Paraná. Rev. Tropical Plant Pathology., 35(5), 295-302.

Kantolic, G., Giménez, P.I. y De La Fuente, E.B. (2003). Ecofisiología del cultivo de soja; bases para el manejo y para el aumento del rendimiento potencial. En: Reunión de Actualización en Soja Criadero Don Mario, 2 de junio 2003. Trabajos presentados. Buenos Aires: Facultad de Agronomía. Cátedra de Cultivos Industriales. Departamento de Producción Vegetal, 6 p.

Leiva, N.P.F., Severino, J.J. é Zabini, A.V. (2016). Ocorrência de fitonematoides na região oriental do Paraguai. Em: $33^{\circ}$ Congresso Brasileiro de Nematologia. 12 a 17 de junio de 2016. Petrolina, Brasil: EMBRAPA

McCarter, J.P. (2009). Molecular approaches toward resistance to plant-parasitic nematodes. In: Berg, R.H., Taylor, C. Cell Biology of Plant Nematode Parasitism: Springer. Berlin Heidelberg, 239-267.

Monteiro, A.R. \& Lordello, L.G.E. (1980). Tubixaba tuxaua n.gen. n.sp. A suspected parasitic nematode of soybean roots (Aporcelaimidae). Rev. de Agricultura, 55(4), 301-304.

Niblack, T.L. (2005). Soybean cyst nematode management reconsidered. Plant disease, 89(10), 1020-1026.

Niblack, T.L. (2009). Nematode. In. Illinois Agronomy Handbook, 24th Edition: University of Illinois at Urbana-Champaign, College of Agriculture, Cooperative Extension Service, USA, 209- 218.

Roese, A.D., Romani, R.D., Furlanetto, C., Stangarlin, J.R. é Portz, R.L. (2001). Levantamento de doenças na cultura da soja em municípios da região oeste do estado do Paraná. Acta Scientiarum (BR), 23, 1293-1297.

Sikora, R.A., Bridge, J. \& Starr, JL. (2005). Management Practices: an Overview of Integrated Nematode Management Technologies. In: Luc, M; Sikora, RA; Bridge, J. Plant Parasitic Nematodes in Subtropical and Tropical Agriculture. 2 ed: CAB International, Wallingford, UK, 793-825.

Tihohod, D. (1993). Nematología Agrícola Aplicada. Jaboticabal: FUNEP, pp. 372.

Vovlas, N., Lamberti, F., Antonio, H., Monteiro, A.R. \& Sharma, R.D. (1987). Morphological characteristics of Tubixaba tuxaua Monteiro \& Lordello (Nematoda: Aporcelamidae). Rev. Nematologia Brasileira, 11, 293-299. 